\title{
Análisis del grupo sanguíneo Rh en fratrias. ¿Un nuevo sistema de histocompatibilidad en el hombre?
}

\author{
Carlos Y. Valenzuela, ${ }^{1}$ Alffedo Avendaño B., ${ }^{2}$ Zuraiya Harb D. ${ }^{1}$ y Mónica Acuña P.'
}

\begin{abstract}
The Rh blood system is examined in a sample of children from the Northem Area of Santiago. AntiC, unthe. antiD, antiE and antie antisera are used. 73 sib-sib pairs were included with the condition that brothers or sisters had no more than three years of difference in age in relation to their respective index case. lndex cases presented higher frequencies of RH3 (CDe/CDe) and lower frequencies of RH5 (CDe/cDE) and RH6 (CDe/cde) than brothers or sisters. From 17 sib-sib pairs were the index subject was RH3, only one sib was also RH3, the remainder sibs were RH5 (3) RH6 (11) RH7 (cDE/cDE, 1) and RHIb (cdejcdel). The probability of random production of this distribution is $\mathrm{P}<0.00002$. We postulate that a major hystocompatiblity system is associated to the Ph system in the hurnan chromosome 1 in order to explain our results.
\end{abstract}

En un trabajo previo describimos los parámetros principales de algunos grupos sanguíneos tipificados en una muestra de escolares del Area Norte de Santiago que están participando en un estudio de seguimiento longitudinal. ${ }^{1}$ En ese trabajo concluimos que la muestra examinada no representaba a una población panmictica, sino más bien a una población en vías de miscegenación. Concluimos además que había ciertas desviaciones de la segregación del cromosoma 9 en relación con el sexo. No examinamos detalladamente todas las posibles interacciones entre los diferentes grupos pues se necesitarán varios trabajos para realizarlo. Una de las anomalias mencionadas fue que el grupo RH3 que corresponde mayoritariamente al genotipo CDe/CDe presentaba en los varones una alta frecuencia del grupo sanguíneo $O$ del sistema $\mathrm{ABO}_{\text {; }}$ esta frecuencia era sigtificativamente distinta de la encontrada en el total de los varones. Nuestro siguiente análisis corresponde a las relaciones del sistema Rh en las fratras.

\section{POBLACION Y METODO}

La muestra corresponde a escolares del Area Norte de Santiago, escogidos aleatoriamente de los ingresados a Enseñanza Básica en 1973. Se solicitó la concurrencia de los hermanos que no

\footnotetext{
${ }^{1}$ Departamento de Biologia Celular y Cenética y 2 Departamento de Pediatria, Facultad de Medicina, Santiago Norte, Lniversidad de Chile. Area Norte de Sautiago.

Este trabajo hu sido financiado por recursos ondinarios del S.N.S. y de la Universidad de Chile, parcialmente por los Proyectos 3097 1975), MU50-705 M050-801, M797-801 (1980) del Servicio de Desarrollo Cientifico, Creación Artística y Cooperación Intemacional de la Universidad de Chile, y por elConvenio Linidad de Genética Humana (CECOM).
}

tuvieran más de tres años de diferencia con los casos índices seleccionados. En 1977 se empezó un estudio de variables sanguíneas, entre las cuales se incluyó el sistema sanguineo $R$ hue se tipificó con antiC, antic, antiD, antiE y antie de los laboratorios DADE y HYLAND y con suero de Coombs de PFIZER. Otras especificaciones de la muestra están en los trabajos previos. 1,2

La nomenclatura, los tenotipos con sus respectivos genotipos más probables se dan en la Tabla 1.

El cálculo de los genotipos más probables se ha hecho aceptando las frecuencias génicas publicadas en un estudio realizado en la misma Area Norte de Santiago ${ }^{3}$ y aceptando que la población se encuentra en equilibrio de Hardy-Weinberg. Las probabilidades de encontrar un hermano de un fenotipo Rh dado que el índice es de un determinado fenotipo $\mathrm{Rh}$ se encontraron aplicando un programa especialmente diseñado por uno de los autores ${ }^{4}$ de acuerdo con el método de las matrices ITO. ${ }^{5}$

\section{RESULTADOS}

La Tabla 1 que es un resumen del trabajo previo presenta la distribución fenotípica según sexo $y$ tipo de sujeto. Tabla 2.

Se observa que mientras RH3, RH7 y RH8 tienen frecuencias superiores en los indices, RH5 y RH6 se dan más frecuentemente en los hermanos. Estas diferencias son más marcadas en los varones que en las mujeres. La diferencia más notable es la del grupo RH3 en los varones; la probabilidad de esta diferencia calculada con test de $\mathrm{X}^{2}$ y con corrección de Yates es $\mathbf{P}<0.05$. Esta significación está subestimada pues se trata de hemanos que 
Tabla 1.

Nomenclatura, fenotıpos y genotipos más probables del sistema $\mathbf{R h}$

\begin{tabular}{|c|c|c|c|c|c|}
\hline \multirow[t]{2}{*}{ Nombre } & \multicolumn{4}{|c|}{ Fenotipo } & \multirow{2}{*}{ Genotipo Más probable } \\
\hline & C & c $D$ & $\mathbf{E}$ & e & \\
\hline FHI & + & -+ & + & - & $\mathrm{CDE} / \mathrm{CDE}(\mathrm{Hom}) *$ \\
\hline RH2 & + & + & $T$ & + & $\mathrm{CDE} / \mathrm{CDe}$ (Het) \\
\hline RH3 & + & -+ & - & + & CDe/CDe $(\mathrm{Hom})$ \\
\hline RH4 & + & ++ & + & - & $\mathrm{CDE} / \mathrm{CDE}$ (Het) \\
\hline RH5 & + & ++ & + & - & CDe/cDE (Het) \\
\hline RH6 & + & ++ & - & + & CDe/cde (Het) \\
\hline KH7 & - & ++ & + & - & CDE/cDE (Hom) \\
\hline RH४ & - & ++ & + & + & $\mathrm{cDE} / \mathrm{cde}(\mathrm{Het})$ \\
\hline RH9 & - & ++ & - & + & $\mathrm{cDE} / \mathrm{cde}$ (Het) \\
\hline RH18 & - & + & - & + & cdeicde (Hon') \\
\hline
\end{tabular}

*Hom = hamoxigoto, Her $=$ hetenocigoto

Tabla 2

Distribución fenotipica para el sistema hh segin sexo y ulasificación del sujeto

\begin{tabular}{|c|c|c|c|c|c|c|c|c|}
\hline \multirow[t]{3}{*}{ Fenotipo } & \multicolumn{4}{|c|}{ Varones } & \multicolumn{4}{|c|}{ Mujeres } \\
\hline & \multicolumn{2}{|c|}{ Indices } & \multicolumn{2}{|c|}{ Hermanos } & \multicolumn{2}{|c|}{ Indices } & \multicolumn{2}{|c|}{ Hermanas } \\
\hline & N. 0 & $\%$ & N. ${ }^{\circ}$ & $\%$ & N. ${ }^{\circ}$ & $\%$ & N. 0 & $\%$ \\
\hline PHJ & 2 & 1.35 & 0 & 0.00 & 0 & 0.00 & 0 & 0.00 \\
\hline $\mathrm{HH} 2$ & 0 & 0.00 & 1 & 2.70 & 1 & 0.64 & 0 & 0.00 \\
\hline RH3 & 43 & 29.05 & 4 & 10.81 & 36 & 23.08 & 9 & 20.00 \\
\hline RH4 & 2 & 1.35 & 0 & 0.00 & 4 & 2.56 & 0 & 0.00 \\
\hline HH5 & 28 & 18.92 & $1 \mathrm{~J}$ & 29.73 & 41 & 26.28 & 13 & 28.29 \\
\hline RH 6 & 37 & 25.00 & 14 & 37.83 & 40 & 25.64 & 14 & 31.11 \\
\hline RH7 & 10 & 6.76 & 2 & 5.41 & 15 & 9.62 & 4 & 8.89 \\
\hline KH8 & 14 & 9.46 & 3 & 8.11 & 10 & 6.41 & 2 & 4.44 \\
\hline RH9 & 4 & 2.70 & 0 & 0.00 & 2 & 1.28 & 0 & 0.00 \\
\hline RH18 & 8 & 5.41 & 2 & 5.41 & 7 & 4.49 & 3 & 6.67 \\
\hline Total & 148 & & 37 & & $1 \tilde{6} 6$ & & 45 & \\
\hline
\end{tabular}

Tabla 3

Distribución de grupos Rh en fratrias

Hermano(A)

Indice

$\begin{array}{llllllllllll}\text { Rh } & 2 & 3 & 4 & 5 & 6 & 7 & 8 & 9 & 18 & 19 & \text { Total }\end{array}$

Rh

2

3

4

5

6

i

19

Total

$\begin{array}{rrrcccccccr}0 & 0 & 0 & 0 & 0 & 0 & 0 & 0 & 0 & 0 & 0 \\ 0 & 1 & 0 & 3 & 11 & 1 & 0 & 0 & 1 & 0 & 17 \\ 0 & 0 & 0 & 1 & 0 & 0 & 0 & 0 & 0 & 0 & 1 \\ 0 & 2 & 0 & 10 & 1 & 1 & 0 & 0 & 0 & 2 & 16 \\ 1 & 4 & 0 & 4 & 6 & 0 & 1 & 0 & 2 & 0 & 18 \\ 0 & 0 & 0 & 3 & 1 & 1 & 2 & 0 & 0 & 1 & 8 \\ 0 & 0 & 0 & 1 & 1 & 0 & 1 & 0 & 1 & 0 & 4 \\ 0 & 0 & 0 & 0 & 0 & 0 & 1 & 0 & 0 & 0 & 1 \\ 0 & 0 & 0 & 0 & 2 & 1 & 0 & 0 & 1 & 1 & 5 \\ 0 & 1 & 0 & 0 & 1 & 1 & 0 & 0 & 0 & 0 & 3 \\ 1 & 8 & 0 & 22 & 23 & 5 & 5 & 0 & 5 & 4 & 73\end{array}$


tienen una correlación de 0.5 con sus respectivos casos indices. Es obligatorio entonces analizar las fratrías. La Tabla 3 describe ęstas relaciones entre hermanos; el caso indice se describe en las filas y el hernano en las colummas.

Hemos hecho abstracción del sexo para cvitar complicaciones innecesarias y subdivisiones que llevarjan a reducir el número en cada casillero.

A primera vista resalta la fila de $\mathrm{RH} 3$; de 17 casos indices sólo 1 tuvo un hermano RH3, los restantes tuvieron hermanos RH6,(11) RH5,(3) FH7(1) y RH 18,(1) La probabilidad calculada para que este evento suceda por simple azar es $\mathrm{P}<$ 0.00002 . Las atras filas presentan también ciertas anomalías de distribución pero no alcanzan el nivel estadístico de RH3. La probabilidad de obtener 10 o más hermanos RH5 de 14 índices RH5 es $P<0.085$. La probabilidad de obtener 6 o menos henmanos FH6 de 18 indices PH6 es $\mathrm{P}<0.13$. La probabilidad de obtener 1 o menos hermanos RH7 de 7 indices HH7 es $P<0.19$. Se puede realizar un análisis conjunto de grupos, pero en ese caso toda significación estará escondida por la dada por el grupo RH3. Análisis más sofisticados se realizarán posteriormente en otros trabajos. Si se calcula la probabilidad de la diferencia de proporciones del grupo RH3 entre indices y hermanos por un test de t de proporciones, ésta resulta ser $P<0.03$; es decir, existe una clara tendencia a disminuir el grupo RH3 en los hermanos.

\section{DISCUSION}

EI resultado más Ilamativo corresponde a la posible fuerza selectiva que estaria operando en los matrimonios que han producido un hijo RH3 y que por razones desconocidas no vuelven a repetir (con la excepción de 1 caso en 17) el mismo fenotipo. La hipótesis que nos parece más plausible es la existencia de un sistema de histocompatibilidad asociado en el cromosona I al gripo Rh. Las evidencias para que no sea el mismo sistema Rh se dan al repetirse las mismas especificidades antigénicas en los hermanos de los grupos RH6, RH5 y RH7, grupos que al parecer son bien tolerados. Creemos que podría tratarse de un sistema de histocompatibilidad que estaria achuando en las primeras etapas de la vida embrionaria, dado que los hermanos seleccionados no pueden tener más de tres años de diferencia en edad. Otra hipótesis que puede postularse es la existencia de algún gropo Rh escondido o producido por alguna interacción no descubierta aún; llama la atención en este sentido que todos los hernanos, a excepción del que tenia el vrupo RH3, poseían el antígeno $\mathrm{c}$. Hay aún otras hipótesis más complejas pero es mis productivo empezar a trabajar de tal modo de confirmar o descartar las que aparecen más plausibles a primera vista.

\section{AGRADECIMIENTOS}

A la Dra. Mireya Bravo y a todo el personal de los Iaboratorios del Hospital Roberto del Rio, como al personal del CECOM sea nuestoo agradecimiento por hacer posible este trabajo. A la SJa. Nancy Muñoz del CNCI que revisó pacientemente cada una de las fichas.

\section{RESUMEN}

Se examina el sistema sanguíneo Rh en una muestra dé niños del Area Norte de Santiago. Los antisueros utilizados fueron: antiC, antic, antiD, antiE y antie 73. parejas de hennanos quedaron incluidas en la muestra, los hemamos no tienen más de tres años de diferencia en edad que sus respectivos casos indices. Los casos índices presentaron frecuencias superiores de $\mathrm{RH} 3$ (CDe/CDe) e inferiores de RH5 (CDe/cDE) y RH6 (CDe/cde) que las encontradas en la muestra de hermanos. De 17 parejas de hermanos donde los indices pertenecian al grupo RH3, sólo un hemmano tuvo ese mismo fenotipo, el resto se distribuyó en: RH5,(3) RH6,(11) RH7 (cDE/cDE, 1) y RH18 (cde/cde, 1). La probabilidad de producción al azar de este resultado es $P<0.00002$. Para explicar estos resultados, postulamos que hay un sistema mayor de histocompatibilidad asociado al sistema Rh en el cromosoma 1 humano.

\section{REFERENCIAS}

IValentriela C.r., Alendaño, A., Harb, Z. y Acuña, M. Grupos sargunineos en escolares de un seguimiento longitudinal: un cxtruño serendipismo. (Enviado a Rer. Chilena de Ped.).

Acendario, A., Valenatuelti, C.Y., Figiteroa, L. y Col. Estudio lonpitudinal del creciniento y desarnilo de un $10 \%$ de los niños que ingresan a la Enseranza Básica Fiscal, Ared Hospitalatira Norte de Santiago. Pedlatria. 19, 2, 156-159, 1976.

3 Armanet, L., Ling, C.. Correa, N., Islas, E., Vutenzurta, C.1.y Hitrl, Z. Frecuencia de lus sistemas Rh, MMSs, Duffy, Diego, Kell, Lutheran y $\mathrm{X}_{\mathrm{g}}$ en Chile. Rer. Mett. Chile 105: It, 1 H66, 1980.

4Valenzuela, C.Y. Un programa para obtener las matrices de probabiliclades de relaciones entre bemanos. Aplicución al caso de gemelos. (Aveptado en Fev, Med. Chile).

${ }^{5} \mathrm{Ll}, \mathrm{C}, \mathrm{C}$. First course in Population Genetics. Pacitic Grove. Califorria. The Boxwood Yress. 1976. 